\title{
Feynman Formulas for Evolution Equations with Levy Laplacians on Infinite-Dimensional Manifolds
}

\author{
L. Accardi* and O. G. Smolyanov** \\ Presented by Academician V.S. Vladimirov October 20, 2005
}

Received October 21, 2005

\section{DOI: $10.1134 /$ S106456240602027X}

A Feynman formula is a representation of the solution to the Cauchy problem for an evolution partial differential (or pseudodifferential) equation in terms of the limit of a sequence of multiple integrals with multiplicities tending to infinity. The integrands are products of the initial condition and Gaussian (or complex Gaussian) exponentials ${ }^{1}$ [5]. In this paper, we obtain Feynman formulas for the solutions to the Cauchy problems for the Schrödinger equation and the heat equation with Levy Laplacian on the infinite-dimensional manifold of mappings from a closed real interval to a Riemannian manifold. The definition of the Levi Laplacian acting on functions on such a manifold is obtained by combining the methods of papers [3] and [7]. In the former, Levi Laplacians in the space of functions on an infinitedimensional vector space were considered, and in the latter, Volterra Laplacians in the space of functions on the above infinite-dimensional manifold were examined. This definition of a Levi Laplacian is equivalent to that given in [2], but it is better adapted for derivation of Feynman formulas.

The main idea of the proof of the central result of this paper is reducing the derivation of Feynman formu-

\footnotetext{
${ }^{1}$ For the heat equation, these multiple integrals coincide with integrals being finite-dimensional approximations to integrals with respect to the Wiener measure. For the Schrödinger equation, such integrals coincide with those used in the definition (which goes back to Feynman himself) of sequential Feynman path integrals. Therefore, the limits of multiple integrals in the Feynman formulas are integrals with respect to the Wiener measure in the former case and (sequential) Feynman path integrals in the latter case, and in both cases, the Feynman-Kac-type formulas are consequences of the Feynman formulas being discussed.
}

\footnotetext{
* Volterra Mathematical Center, Department of Mathematics, The Second University of Rome "Tor Vergata," Rome, 00173 Italy

** Moscow State University, Leninskie gory, Moscow, 119992 Russia

e-mail:smolyanov@yandex.ru
}

las for equations on a manifold to the derivation of similar formulas for equations on a vector space. For equations on finite-dimensional manifolds (containing the usual finite-dimensional Laplacians), this approach was suggested in [4] and developed in [6]. The Riemannian manifold under consideration was embedded in a suitable Euclidean space (this can always be done by the Nash theorem), and the technique of surface measures developed in $[4,7]$ was applied. An essential point in the proof was the application of the Chernoff formula (generalizing the Trotter formula), which is related to obtaining representations of solutions to evolution equations on manifolds (and to representations of solutions to equations on vector spaces in terms of path integrals in the phase space [5]) in the same way as the Trotter formula is related to representations of the solution to the simplest Schrödinger equation with potential in terms of path integrals in the configuration space.

The remark made in the footnote means that the results obtained in this paper contain the construction of a Levy Brownian motion on the set of paths on a (compact) Riemannian manifold (cf. [9, 8, 3]). Note also that the interest to equations with Levy Laplacians is largely caused by the fact that the Yang-Mills gauge fields are harmonic functions for the Levy Laplacian (see [1] and references therein).

The exposition in this paper is formal in part; some analytical assumptions are omitted.

\section{PRELIMINARIES AND NOTATION}

For any metric space $E$ and a number $a>0, C([0, a]$, $E$ ) denotes the set of all continuous mappings from the interval $[0, a]$ to $E$ endowed with the uniform metric. For each $x \in E, C_{x}([0, a], E)$ is the subspace of the metric space $C([0, a], E)$ formed by the functions taking the value $x$ at zero. If $E$ is a Riemannian $C^{k}$-manifold, $n \in \mathbb{N}$, and $n \leq k$, then $C^{n}([0, a], E)$ and $C_{x}^{n}([0, a], E)$ are the infinite-dimensional manifolds of $n$-times continuously 
differentiable functions from $C([0, a], E)$ and from $C_{x}([0, a], E)$, respectively. We assume that these manifolds are endowed with the (metrizable) topologies of the uniform convergence of functions and their derivatives up to order $n$. The space $W_{2, x}^{1}([0, a], E)$, where $x \in E$, is defined as follows. Let $E$ be a Riemannian $C^{j}$-submanifold $(j \geq 2)$ in $\mathbb{R}^{k}$, and let $W_{2}^{1}\left([0, a], \mathbb{R}^{k}\right)$ be the space of all $\mathbb{R}^{k}$-valued absolutely continuous functions on $[0, a]$ vanishing at zero and having square-integrable derivatives with the (Hilbert) norm defined by

$$
\|g\|^{2}=\int_{0}^{a}\left\|g^{\prime}(t)\right\|_{\mathbb{R}^{k}}^{2} d t \text {. For every } z \in E, \text { let } g_{z} \in C([0, a], E)
$$

be the function defined by $g_{z}(t)=z$ for $t \in[0, a]$. Then, $W_{2, z}^{1}([0, a], E)=\left(W_{2}^{1}\left([0, a], \mathbb{R}^{k}\right)+g_{z}\right) \cap C([0, a], E)$. This definition does not depend on the embedding of $E$ into $\mathbb{R}^{n}$ [on the space $W_{2, z}^{1}([0, a], E)$ [which contains $\supset$ $C_{z}^{n}([0, a], E)$ for $\left.n \geq 1\right]$, there is the natural structure of a Hilbert manifold generated by the Hilbert space structure on $W_{2}^{1}\left([0, a], \mathbb{R}^{k}\right)$, but we do not use it in what follows $]$. The space $W_{2, q}^{1}\left([0, t],\left(W_{2, z}^{1}([0, a], G)\right)\right.$, where $q \in W_{2, z}^{1}([0, a], G)$, is defined similarly.

Let $E$ be a Banach space, and let $H$ be its Hilbert subspace; this means that $H$ is a vector subspace of $E$ and the canonical embedding of $H$ into $E$ is continuous. If $B$ is a continuous positive definite operator on $H$, then the cylindrical Gaussian measure on $E$ with $(H-)$ correlation operator $B$ is defined as the image (under the canonical embedding of $H$ into $E$ ) of the cylindrical Gaussian measure on $H$ with correlation operator $B$. Under the same assumptions, a (cylindrical) Wiener process on $[0, a]$ taking values in $E$, or a (cylindrical) Brownian motion in $E$ (defined on $[0, a]$ ), starting at $x \in E$ and having correlation operator $B$ is defined as a (cylindrical) homogeneous Markov process in $E$ that starts at $x \in E$, is defined on $[0, a]$, and has transition probability $\mathrm{P}^{B}(t, x, \cdot)=\mathrm{P}^{E}(t, \cdot)+x$, where $t>0, x \in E$, and $\mathrm{P}^{E}(t, \cdot)$ is the cylindrical Gaussian measure on $E$ with $H$-correlation operator $t B$ and mean zero. The cylindrical probability on $C([0, a], E)$ generated by this process is called a Wiener measure with $(H$-)correlation operator $B$; it can be assumed to be concentrated on $C_{x}([0, a]$, $E)$. Moreover, $\mathrm{P}(\cdot, \cdot, \cdot)$ is the integral kernel of the solving operator of the Cauchy problem for the heat equation on $E$ with the Laplace operator $\Delta^{B}$ defined by $\left(\Delta^{B} g\right)(x)=\operatorname{tr}\left(B g^{\prime \prime}(x)\right.$, where the symbol $g^{\prime \prime}(x)$ denotes the second derivative (along $H$ ) of the function $g$ and $\operatorname{tr}$ denotes the trace of the corresponding operator on $H$ $\left(\Delta^{B}\right.$ is sometimes called the Volterra Laplacian generated by $B)$.

\section{DOKLADY MATHEMATICS Vol. 73 No. 2 2006}

\section{LAPLACE OPERATORS}

In this section, we define the Laplace-Volterra operator $\Delta_{V}$ and the Laplace-Levy operator $\Delta_{L}$ on the spaces of functions on $W_{2, x}^{1}([0, a], G)$, where $x \in G$ and $G$ is a Riemannian manifold (although the main purpose of this paper is studying equations with Levy Laplacian, it is useful to define both operators simultaneously). In what follows, we assume that the manifold $G$ is either the entire Euclidean space or a compact space.

Suppose that $G$ is a $C^{k}$-submanifold with $k>2$ of dimension $d$ in $\mathbb{R}^{n}, F$ is a function on $W_{2, x}^{1}([0, a], G)$, and $\psi \in C_{x}^{2}([0, a], G)$. For each $t \in[0, a]$, let $\left\{z_{1}^{t}\right.$, $\left.z_{2}^{t}, \ldots, z_{d}^{t}\right\}$ be an orthonormal basis in the space tangent to $G$ at $\psi(t)$ obtained by translating some fixed (not depending on $t$ ) orthonormal basis in the space tangent to $G$ at $x$ along $\psi([0, t])$. For each $r=1,2, \ldots, d$, we use $c_{r}^{t}$ to denote the geodesic passing through $\psi(t)$ in the direction of the vector $z_{r}^{t}$. Suppose also that $\left\{e_{n}^{V}\right\}$ is an orthonormal basis in $W_{2}^{1}\left([0, a], \mathbb{R}^{1}\right)$. For integers $k=1$, $2, \ldots, d$ and $p \in \mathbb{N}$ and a number $\alpha \in \mathbb{R}$ with sufficiently small absolute value, let $\psi_{\alpha}^{k, p} \in C_{x}^{1}([0, a], G)$ be the function defined as follows: $\psi_{\alpha}^{k, p}(t)$ is the element of the geodesic $c_{k}^{t}$ such that the distance from this element to $\psi(t)$ along $c_{k}^{t}$ equals $\left|\alpha e_{p}^{V}(t)\right|$ and the direction from $\psi(t)$ to this element coincides with that of $z_{k}^{t}$ if $\alpha e_{p}^{V}(t)>0$ and is opposite to that of $z_{k}^{t}$ if $\alpha e_{p}^{V}(t)<0$. For the same $k$ and $p$, we define a real function $F_{k, p}^{\psi}$ of the argument $\alpha$ by the equality $F_{k, p}^{\psi}(\alpha)=F\left(\psi_{\alpha}^{k, p}\right)$.

Definition 1. A function $F: W_{2, x}^{1}([0, a], G) \rightarrow \mathbb{R}$ is contained in the domain dom $\Delta_{V}$ of the Laplace-Volterra operator $\Delta_{V}$ if and only if, for each function $\psi \in$ $C_{x}^{2}([0, a], E)$, the series $\sum_{k, p}\left(F_{k, p}^{\psi}\right)^{\prime \prime}(0)$ converges and the function $C_{x}^{2}([0, a], E) \ni \psi \mapsto \sum_{k, p}\left(F_{k, p}^{\psi}\right)^{\prime \prime}(0)$ admits a continuous extension over the space $W_{2, x}^{1}([0, a], G)$. If $F \in \operatorname{dom} \Delta_{V}$, then $\Delta_{V} F$ is this continuous extension.

Remark 1. A Brownian motion (Wiener process) in $C([0, a], G)$ starting at $q \in C([0, a], G)$ can be defined as a homogeneous Markov process in $C_{q(0)}([0, a], G)$ starting at $q$ with transition probability coinciding with the Green measure of the heat equation with LaplaceVolterra operator $\Delta_{V}$. Let $\mathcal{W}_{q, t}$ be the corresponding 
Wiener measure on $C_{q}\left([0, t], C_{q(0)}([0, a], G)\right)$. It can be shown that the measure $\mathcal{W}_{q, t}$ coincides with the surface measure generated by the (Wiener) measure on $C_{q}([0$, $\left.t], C_{q(0)}\left([0, a], \mathbb{R}^{n}\right)\right)$ that is a translation of the Wiener measure on $C\left([0, t], C\left([0, a], \mathbb{R}^{n}\right)\right)$ whose $W_{2}^{1}([0, a]$, $\mathbb{R}^{n}$ )-correlation operator coincides with the identity operator on $W_{2}^{1}\left([0, a], \mathbb{R}^{n}\right)$.

In what follows, we identify the spaces $C\left(\left[0, a_{1}\right]\right.$, $\left.C\left(\left[0, a_{2}\right], G\right)\right)$ and $C\left(\left[0, a_{1}\right] \times\left[0, a_{2}\right], G\right)$.

Suppose that $\left\{e_{n}^{L}\right\}$ is an orthonormal basis in $L_{2}([0$, $\left.a], \mathbb{R}^{1}\right),\left\{e_{n}^{L}\right\} \subset C^{2}\left([0, a], \mathbb{R}^{1}\right)$, and $\varphi \in C_{x}^{2}([0, a], G)$. For integers $k=1,2, \ldots, d$ and $p \in \mathbb{N}$ and a number $\alpha \in \mathbb{R}$ with sufficiently small absolute value, we define a function $\varphi_{\alpha}^{k, p} \in C_{x}^{1}([0, a], G)$ as follows: $\varphi_{\alpha}^{k, p}(t)$ is the element of the geodesic $c_{k}^{t}$ such that the distance from $\varphi(t)$ (along $c_{k}^{t}$ ) to this element equals $\left|\alpha e_{p}^{L}(t)\right|$ and the direction from $\varphi(t)$ to this element coincides with that of $z_{k}^{t}$ if $\alpha e_{p}^{L}(t)>0$ and is opposite to that of $z_{k}^{t}$ otherwise. For $F: W_{2, x}^{1}([0, a], G) \in \mathbb{R}$, we define a function $F_{k, p}^{\varphi, L}: \mathbb{R} \rightarrow \mathbb{R}$ by setting $F_{k, p}^{\varphi, L}(\alpha)=F\left(\varphi_{\alpha}^{k, p}\right)$.

Definition 2. A function $F: W_{2, x}^{1}([0, a], G) \rightarrow \mathbb{R}$ is contained in the domain $\operatorname{dom} \Delta_{L}$ of the Laplace-Levy operator $\Delta_{L}$ if and only if, for each function $\varphi \in C_{x}^{2}([0, a]$, $E)$, the limit $\lim _{r \rightarrow \infty} \frac{1}{d r} \sum_{k=1, p=1}^{k=d, p=r}\left(F_{k, p}^{\varphi, L}\right)^{\prime \prime}(0)$ exists and the function $C_{x}^{2}([0, a], E) \ni \psi \mapsto \lim _{r \rightarrow \infty} \frac{1}{d r} \sum_{k=1, p=1}^{k=d, p=r}\left(F_{k, p}^{\varphi, L}\right)^{\prime \prime}(0)$ admits a continuous extension over the space $W_{2, x}^{1}$ ([0, $a], G)$. If $F \in \operatorname{dom} \Delta_{L}$, then $\Delta_{L} F$ is this continuous extension.

Remark 2. For $G=\mathbb{R}^{k}$, the definitions of the Levy and Volterra Laplacians given above coincide with the classical definitions (see, e.g., [3, 8, 9] and the references therein).

\section{FEYNMAN FORMULAS}

Let $\rho$ be the distance on a Riemannian manifold $G$ generated by its Riemannian structure, and let $\operatorname{scal}(q)$ and $m(q)$ be the scalar and (vector-valued) mean curvatures of the manifold $G$ at the point $q \in G$. We assume that $G$ is a Riemannian $C^{k}$-submanifold $(k \geq 3)$ of some Euclidean space with norm $\|\cdot\|$.
Consider the nonnegative functions $p_{R}, Q_{R}, p_{E}$, and $Q_{E}$ on $[0, \infty) \times G \times G$ defined by

$$
\begin{aligned}
& p_{R}\left(t, q_{1}, q_{2}\right)=\exp \left(-\frac{\left(\rho\left(q_{1}, q_{2}\right)\right)^{2}}{2 t}\right) ; \\
& Q_{R}\left(t, q_{1}, q_{2}\right)=\frac{p_{R}\left(t, q_{1}, q_{2}\right)}{\int_{G} p_{R}\left(t, q_{1}, q_{2}\right) d q_{2}} ; \\
& p_{E}\left(t, q_{1}, q_{2}\right)=\exp \left(-\frac{\left\|q_{2}-q_{1}\right\|^{2}}{2 t}\right) ; \\
& Q_{E}\left(t, q_{1}, q_{2}\right)=\frac{p_{E}\left(t, q_{1}, q_{2}\right)}{\int_{G} p_{E}\left(t, q_{1}, q_{2}\right) d q_{2}} ; \\
& p_{E}^{F}\left(t, q_{1}, q_{2}\right)=\exp \left(\frac{i\left\|q_{2}-q_{1}\right\|^{2}}{2 t}\right) ; \\
& Q_{E}^{F}\left(t, q_{1}, q_{2}\right)=\frac{p_{E}^{F}\left(t, q_{1}, q_{2}\right)}{\int_{G}^{F} p_{E}^{F}\left(t, q_{1}, q_{2}\right) d q_{2}} .
\end{aligned}
$$

In what follows, we assume that $a, t>0 ; F$ is the function on $C([0, t] \times[0, a], G)$ defined by $F(g)=$ at

$\iint_{0} V\left(g\left(t_{1}, t_{2}\right)\right) d t_{1} d t_{2}$, where $V$ is a continuous function on $G$; and $q \in W_{2, z}^{1}([0, a], G)$ [thus, $\left.q(0)=z\right]$.

Proposition 1. On the space $W_{2, q}^{1}\left([0, t], W_{2, q(0)}^{1}([0\right.$, a], $G)$ ), there exists a cylindrical probability $\mathcal{W}_{q}^{L}$ (we call it the Levy-Wiener probability) for which

$$
\begin{aligned}
& \int_{W_{2, q}^{1}\left([0, t], W_{2, q(0)}^{1}([0, a], G)\right)} F(q) W_{q}^{L}(d q) \\
= & \lim _{p \rightarrow \infty} \int_{G \times G \times \ldots \times G}\left(\sum_{n=1, k=1}^{p, p} \frac{t_{a}}{p^{2}} V\left(q_{n k}\right)\right) \\
\times & \prod_{n=1}^{p} \prod_{k=1}^{p} Q_{R}\left(p^{-1} t, q_{n, k-1}, q_{n, k}\right) d q_{n, k},
\end{aligned}
$$$$
\text { where } q_{n, 0}=q\left(\frac{n}{p} a\right) \text {. }
$$

The proposition obtained from Proposition 1 by replacing $Q_{R}$ by $Q_{E}$ is also valid.

Remark 3. In Proposition 1 and the subsequent propositions, the choice of $W_{2, q(0)}^{1}([0, a], G)$ as the domain on which the solutions are defined and the cor- 
responding choice of the domain of integration are not very essential (cf. [8]).

The following theorems are concerned with Feynman formulas for the semigroups determining solutions to the Cauchy problems for the heat equation and the Schrödinger equation with Levy Laplacian on the space of functions on $W_{2, z}^{1}([0, a], G)$. The methods and results of [7] make it possible to obtain similar results for the Volterra Laplacian.

Let $\Phi$ be the function on $C([0, a], G)$ defined by

$$
\Phi(g)=\int_{0}^{a} V_{\Phi}\left(g\left(t_{1}\right)\right) d t_{1},
$$

and let $\psi$ be the function on $C([0, a], G)$ defined by

$$
\psi(g)=\int_{0}^{a} V_{\psi}\left(g\left(t_{1}\right)\right) d t_{1},
$$

where $V_{\Phi}$ and $V_{\psi}$ are continuous functions on $G$; suppose that $\hat{H}_{L} f=-\frac{1}{2} \Delta_{L} f+\Phi f$ for any function $f$ : $W_{2, z}^{1}([0, a], G) \rightarrow \mathbb{R}^{1}$. In Theorems 1 and 2 and Propositions 2 and 3 of the next section, it is assumed that the Cauchy problem for the equation $\frac{\partial f}{\partial t}=-\hat{H}_{L} f$ in $[0, \infty) \times$ $W_{2, z}^{1}([0, a], G)$ with initial data $(0, \psi)$ has a solution; we denote the value of this solution at $(t, q) \in(0, \infty) \times$ $W_{2, z}^{1}([0, a], G)$ by $e^{t \hat{H}_{L}} \psi(q)$. In Theorem 3 , it is assumed that the Cauchy problem for the equation $i \frac{\partial f}{\partial t}=$ $\hat{H}_{L} f$ in $[0, \infty) \times W_{2, z}^{1}([0, a], G)$ with initial data $(0, \psi)$ has a solution; we denote the value of this solution at $(t, q) \in(0, \infty) \times W_{2, z}^{1}([0, a], G)$ by $e^{i t \hat{H}_{L}} \psi(q)$.

Theorem 1. If $(t, q) \in(0, \infty) \times W_{2, z}^{1}([0, a], G)$, then

$$
\begin{aligned}
& e^{t \hat{H}_{L}} \psi(q)=\lim _{p \rightarrow \infty} \int_{G \times G \times \ldots \times G} \exp \left\{\frac{t a}{p^{2}} \sum_{n=1, k=1}^{p, p} V_{\Phi}\left(q_{n k}\right)\right. \\
& \times\left(\frac{a}{p} \sum_{n=1}^{n} V_{\psi}\left(q_{n p}\right)\right) \prod_{n=1}^{p} \prod_{k=1}^{p} Q_{R}\left(\frac{t}{p^{2}}, q_{n, k-1}, q_{n, k}\right) d q_{n, k},
\end{aligned}
$$

where $q_{n, 0}=q\left(\frac{n}{p} a\right)$.

This theorem is derived from Proposition 1 with the use of the Chernoff theorem.

The proof of the following theorem uses Proposition 1, the bounds for integrals over Riemannian manifolds obtained in [4], and the Chernoff theorem.
Theorem 2. For all $(t, q) \in(0, \infty) \times W_{2, z}^{1}([0, a], G)$, the following equalities hold [in these equalities,

$$
\begin{aligned}
& \left.q_{n, 0}=q\left(\frac{n}{p} a\right)\right]: \\
& e^{t \hat{H}_{L}} \psi(q)=\lim _{p \rightarrow \infty} c_{p}^{1} \int_{G \times G \times \ldots \times G} \exp \left\{\frac{t a}{p^{2}} \sum_{n=1, k=1}^{p, p} V_{\Phi}\left(q_{n k}\right)\right\} \\
& \quad \times\left(\frac{a}{p} \sum_{n=1}^{p} V_{\psi}\left(q_{n 0}\right)\right) \prod_{n=1}^{p} \prod_{k=1}^{p} \exp \left\{\frac{1}{6} \frac{t}{p^{2}} \operatorname{scal}\left(q_{n, k}\right)\right\} \\
& \quad \times p_{R}\left(\frac{t}{p^{2}}, q_{n, k-1}, q_{n, k}\right) d q_{n, k},
\end{aligned}
$$

where

$$
\begin{gathered}
\left(c_{p}^{1}\right)^{-1}=\int_{G \times G \times \ldots \times G} \prod_{n=1}^{p} \prod_{k=1}^{p} p_{R}\left(\frac{t}{p^{2}}, q_{n, k-1}, q_{n, k}\right) d q_{n, k} ; \\
e^{t \hat{H}_{L}} \psi(q)=\lim _{p \rightarrow \infty} c_{p}^{2} \int_{G \times G \times \ldots \times G} \exp \left\{\frac{t a}{p^{2}} \sum_{n=1, k=1}^{p, p} V_{\Phi}\left(q_{n k}\right)\right\} \\
\times\left(\frac{a}{p} \sum_{n=1}^{p} V_{\psi}\left(q_{n 0}\right)\right) \prod_{n=1}^{p} \prod_{k=1}^{p} \exp \left\{\frac { t } { 4 p ^ { 2 } } \left(\operatorname{scal}\left(q_{n, k}\right)\right.\right. \\
\left.\left.\quad-\frac{1}{2}\left\|m\left(q_{n, k}\right)\right\|^{2}\right)\right\} p_{E}\left(\frac{t}{p^{2}}, q_{n, k-1}, q_{n, k}\right) d q_{n, k},
\end{gathered}
$$

where

$$
\left(c_{p}^{2}\right)^{-1}=\int_{G \times G \times \ldots \times G^{n}=1} \prod_{k=1}^{p} \prod_{E}^{p} p_{E}\left(\frac{t}{p^{2}}, q_{n, k-1}, q_{n, k}\right) d q_{n, k} .
$$

In what follows, we assume that $\sqrt{i}=e^{i \frac{\pi}{4}}$.

Theorem 3. Suppose that $V_{\Phi}$ and $V_{\psi}$ are the restrictions to $G$ of functions (denoted by the same symbols) defined and analytic on a domain of the space $\mathscr{C}^{n}$ that contains $G$ and $\bigcup_{z \in G}\{z+\sqrt{i}(G-z)\}$.

Then, for $(t, q) \in(0, \infty) \times W_{2, z}^{1}([0, a], G)$,

$$
\begin{aligned}
& e^{i t \hat{H}_{L}} \psi(q)=\lim _{p \rightarrow \infty} \int_{G \times G \times \ldots \times G} \exp \left\{\frac{i t a}{p^{2}} \sum_{n=1, k=1}^{p, p} V_{\Phi}\left(q_{n k}\right)\right\} \\
& \times\left(\frac{a}{p} \sum_{n=1}^{p} V_{\psi}\left(q_{n p}\right)\right) \prod_{n=1}^{p} \prod_{k=1}^{p} Q_{E}^{F}\left(\frac{t}{p^{2}}, q_{n, k-1}, q_{n, k}\right) d q_{n, k} .
\end{aligned}
$$


If the Riemannian curvature tensor admits an analytic extension over this domain, then (under the same assumptions)

$$
\begin{aligned}
& e^{i t \hat{H}_{L}} \psi(q)=\lim _{p \rightarrow \infty} c_{p}^{3} \int_{G \times G \times \ldots \times G} \exp \left\{\frac{i t a}{p^{2}} \sum_{n=1, k=1}^{p, p} V_{\Phi}\left(q_{n k}\right)\right\} \\
& \times\left(\frac{a}{p} \sum_{n=1}^{p} V_{\psi}\left(q_{n 0}\right)\right) \\
& \times \prod_{n=1}^{p} \prod_{k=1}^{p} \exp \left\{\frac { t } { 4 p ^ { 2 } } \left(\operatorname { s c a l } \left(q_{n 0}+\frac{1}{\sqrt{i}}\left(q_{n, k}-q_{n 0}\right)\right.\right.\right. \\
& \left.-\frac{1}{2}\left\|m\left(q_{n 0}+\frac{1}{\sqrt{i}}\left(q_{n, k}-q_{n 0}\right)\right)\right\|^{2}\right\} \\
& \times p_{E}^{F}\left(\frac{t}{p^{2}}, q_{n, k-1}, q_{n, k}\right) d q_{n, k}, \\
& \left(c_{p}^{3}\right)^{-1}=\int_{G \times G \times \ldots \times G^{n}=1} \prod_{k=1}^{p} \prod_{E}^{p} p^{F}\left(\frac{t}{p^{2}}, q_{n, k-1}, q_{n, k}\right) d q_{n, k} \\
& {\left[\text { here, } q_{n, 0}=q\left(\frac{n}{p} a\right)\right] \text {. }}
\end{aligned}
$$

This theorem is obtained from Theorem 2 by passing from the Schrödinger equation to the heat equation with the use of the change $q \rightarrow \sqrt{i} q$.

\section{ADDITIONAL REMARKS}

In this section, we suggest yet another method for obtaining approximations of the semigroups generated by the Levy Laplacian. It is based on approximating Levi-Wiener processes by finite-dimensional Brownian motions and substantially differs from that described above. We consider the Laplace-Levy equation first in the space of functions on $W_{2}^{1}\left([0, a], \mathbb{R}^{n}\right)$, and then in the space of functions on $W_{2, x}^{1}([0, a], G)$.

Suppose that $\left\{e_{k}\right\}$ is an orthonormal basis in the space $L_{2}\left([0, a], \mathbb{R}^{n}\right)$ such that $\left\{e_{k}\right\} \subset C^{2}\left([0, a], \mathbb{R}^{n}\right)$; suppose also that, for each $p \in \mathbb{N}, S_{p}$ denotes the linear span of the set $\left\{e_{k}: k=1,2, \ldots, p\right\}$ and $b_{x}^{p}$ denotes the Brownian motion in $S_{p}$ starting at $x \in S_{p}$ whose transition function has correlation operator $\frac{1}{p} \mathrm{Id}$, where Id is the identity self-mapping of $S_{p}$. Finally, suppose that $W_{x, t}^{p}$ with $t>0$ is the Wiener measure on $C_{x}\left([0, t], S_{p}\right)$ generated by the Brownian motion $b_{x}^{p}$ and $\hat{H}_{L} f=\frac{1}{2} \Delta_{I} f+$ $V_{\Phi} f$ for any function $f: W_{2}^{1}\left([0, a], \mathbb{R}^{n}\right) \rightarrow \mathbb{R}^{1}$.
Proposition 2. If $(t, q) \in(0, \infty) \times\left(W_{2}^{1}([0, a], G)\right)$, then

$$
\begin{aligned}
e^{t \hat{H}_{L}} \psi(q)= & \lim _{p \rightarrow \infty} \int_{C_{p(q)\left([0, t], S_{p}\right)}} \exp \left\{\int_{0}^{t} \Phi(\xi(\tau)) d \tau\right\} \\
& \times \psi\left(\xi(t) W_{q(p)}^{p}(d \xi),\right.
\end{aligned}
$$

where $q(p)$ is the orthogonal projection in $L_{2}\left([0, a], \mathbb{R}^{n}\right)$ of the element $q \in W_{2, x}^{1}\left([0, a], \mathbb{R}^{n}\right)$ on $S_{p}$.

The proof uses approximations of the Levy Laplacian by finite-dimensional Laplacians and the perturbation theory of semigroups.

The following proposition is an analogue of Proposition 2 for functions on $W_{2, x}^{1}([0, a], G)$; the symbol $\hat{H}_{L}$ in this proposition denotes the operator defined before the statement of Theorem 1.

Let $S^{G}$ be the linear span of the set $W_{2, x}^{1}([0, a], G)$ in the space $L_{2}\left([0, a], \mathbb{R}^{n}\right)$, and let $\left\{a_{n}\right\}$ be an orthonormal [in the metric of $\left.L_{2}\left([0, a], \mathbb{R}^{n}\right)\right]$ basis of $S^{G}$ consisting of functions from $C^{2}\left([0, a], \mathbb{R}^{n}\right)$ such that, for each $p \in \mathbb{N}$, the dimension of the intersection of the linear span $S_{p}^{G}$ of $\left\{a_{n}: n=1,2, \ldots, p\right\}$ with $C^{2}([0, a], G)$ equals $p$. Suppose that $b_{q}^{p}$, where $q \in S_{p}^{G} \cap C^{2}([0, a], G)$ is the Brownian motion in $S_{p}^{G}$ starting at $q$ whose transition function has correlation operator $\frac{1}{p} \mathrm{Id}$, where Id is the identity self-mapping of the space $S_{p}^{G}$; by $W_{q, t}^{p}$, where $t>0$, we denote the Wiener measure on $C_{q}\left([0, t], S_{p}^{G}\right)$ generated by the Brownian motion $b_{q}^{p}$. Finally, let $W_{G, p, t}^{p}$ be the probability measure on $C\left([0, t], W_{2, x}^{1}\right.$ ( $[0$, $a], G))$ concentrated on the set $C_{q}\left([0, t], S_{p}^{G}\right) \cap C([0, t]$, $\left.W_{2, x}^{1}([0, a], G)\right)$ and coinciding on this set with the surface measure [4] generated by the measure $W_{q, t}^{p}$. then

Proposition 3. If $(t, q) \in(0, \infty) \times\left(W_{2, x}^{1}([0, a], G)\right)$,

$$
\begin{aligned}
e^{t \hat{H}_{L}} \psi(q)= & \lim _{p \rightarrow \infty} \int_{C_{q(p)}\left([0, t], S_{p}^{G}\right)} \exp \left\{\int_{0}^{t} \Phi(\xi(\tau)) d \tau\right\} \\
& \times \psi(\xi(t)) W_{G, q(p), t}^{p}(d \xi),
\end{aligned}
$$

where $q(p)$ is the orthogonal projection $\left[\right.$ in $L_{2}([0, t]$, $\left.\mathbb{R}^{k}\right)$ ] of the element $q$ on $S_{p}^{G}$. 
A similar proposition is valid for the semigroup determining the solution to the Cauchy problem for the Schrödinger equation with Levy Laplacian.

Remark 4. Proposition 3 can also be regarded as a definition of a (cylindrical) Levy-Wiener probability on the space of mappings from the interval $[0, t]$ to $W_{2, x}^{1}([0, a], G)\left[\right.$ or to $\left.C_{x}^{2}([0, a], G)\right]$.

\section{REFERENCES}

1. L. Accardi, P. Gibilisco, and I. V. Volovich, Rus. J. Math. Phys. 2, 235-250 (1994).

2. L. Leandre and I. V. Volovich, Infin. Dimens. Anal. Quantum Probab. Relat. Top. 4 (2), 151-172 (2002).
3. L. Accardi and O. G. Smolyanov, Dokl. Akad. Nauk 384, 295-301 (2002) [Dokl. Math. 65, 356-362 (2002)].

4. O. G. Smolyanov, H. von Weizsaecker, and O. Wittich, Canad. Math. Soc. Conf. Proc. 29, 589-602 (2000).

5. O. G. Smolyanov, A. G. Tokarev, and A. Truman, J. Math. Phys. 43, 5161-5171 (2002).

6. O. G. Smolyanov and A. Trumen, Dokl. Akad. Nauk 392, 174-179 (2003) [Dokl. Math. 68, 194-198 (2003)].

7. O. G. Smolyanov, H. von Weizsacker, and O. Wittich, Dokl. Akad. Nauk 402, 316-320 (2005).

8. L. Accardi and O. G. Smolyanov, Mat. Zametki 64, 483492 (1998).

9. L. Accardi, P. Rozelli, and O. G. Smolyanov, Mat. Zametki 54 (5), 144-149 (1993).

10. R. P. Chernoff, J. Funct. Anal. 40, 239 (1968). 